\title{
Model-based Methodology for Identifying Capacity Limitations in Airports: Mexico City Airport Case
}

\author{
${ }^{1}$ Amsterdam University of Applied Sciences, Aviation Academy, \\ Weesperzijde 190,1097DZ. The Netherlands; *m.mujica.mota@hva.nl \\ ${ }^{2}$ National Autonomous University of Mexico, Mexico City, Mexico
}

Miguel Mujica Mota ${ }^{1 *}$, Idalia Flores ${ }^{2}$

SNE 29(1), 2019, 13 - 25, DOI: 10.11128/sne.29.on.10462

Submitted/Received: November 27, 2018;

Revised: March 21, 2019; Accepted: March 22, 2019;

SNE - Simulation Notes Europe, ARGESIM Publisher Vienna

ISSN Print 2305-9974, Online 2306-0271, www.sne-journal.org

Abstract. The current study presents a methodology for analysing and identifying the limitations in capacity of an airport, the methodology has been implemented in the case of Mexico City Airport which is a congested airport in Mexico. The methodology allows identifying what room is left for absorbing more traffic and what options are available while a new infrastructure is in place. The methodology revealed, that there is still room for absorbing more traffic under certain conditions and starting from that, actions can be taken in order to increase the capacity or reducing congestion in the airport.

\section{Introduction}

Simulation is the imitation of the operation of a real system or process over time. It is used to generate artificial history and data of a system, and the observation and analysis of that artificial history to draw inferences concerning the operating characteristics of the real system (Banks et al. 1996).

The behaviour of a system as it evolves over time is studied by developing a simulation model. This model usually takes the form of a set of assumptions concerning the operation of the system. These assumptions are expressed in mathematical, logical, and symbolic relationships between the entities, or objects of interest of the system. Once developed, verified and validated, a model can be used to investigate a wide variety of "what-if" questions about the real-world system (Banks et al. 1996). Potential changes to the system can then be simulated to predict their impact on the system's performance.

Furthermore, simulation can also be used to study systems in the design stage, before such systems are built based on relationships taken from a similar system.
Thus, simulation techniques can be used both as an analysis tool for analysing and predicting the effect of changes to existing systems, and as a design tool to predict the performance of new systems under varying sets of circumstances. Simulation itself has proven to give good solutions in different fields such as transport industry (Longo, 2011), manufacturing (Latorre et al., 2013) and in some airport problems (Mujica Mota 2015), (Yan et al. 2002) among others.

Simulation's power lies in its capacity of using different abstraction levels and being able to stress many aspects of the problems than cannot be addressed with other techniques such as stochastic behaviour, emergent dynamics or cause-effect relationships. When analysing specific problems such as scheduling or optimization of resources, it cannot ensure the optimal outcome since the experiments performed with the model explore a subset of the whole different configurations of the system under study. However, with the inclusion of uncertainty in the form of probability distributions, we ensure that we consider extreme situations, (that were not part of the data used for the construction of the model) that put in risk the behaviour of the system, also known as tail behaviour (Biller and Nelson 2002).

Furthermore, the outcomes from simulation are more feasible or realistic for being implemented in the real system, which sometimes is not the case of exact solutions coming from traditional analytical techniques. Some authors can argue that with the use of simulation for the decision-making process there is an implicit level of uncertainty, this is correct, however this drawback can be overcome by increasing the number of experiments with the model (Law 2015).

Simulation has been traditionally considered an evaluative tool in which the analysis of systems is performed executing several experiments with the simulation model to obtain insight about the behaviour of the system under study in combination with statistics. 
The simulation models can be developed using different tools that range from general-purpose to specificpurpose ones. The advantage of using a general-purpose tool over a specific one is that it provides the developer or analyst more freedom for including different angles, elements and/or resolution levels of the problem under study. On the contrary, specific-purpose tools are limited to the perspective of the owner/developer of the tool which in most of the cases involves the payment of a commercial license for its use.

For general-purpose tools that can be used for studying aviation-related problems we can mention modelling formalisms such as coloured Petri nets (Jensen \& Kristensen, 2009) or commercial software such as SIMIO (SIMIO 2017) or ARENA (Rockwell 2017). In the case of specific-purpose tools for aviation we can mention ARC's CAST (2015), SIMMOD (ATAC 2017), or TAAM (Jeppesen 2017) among others in which the modeller uses mostly a drag \& drop approach from a library of predefined objects for developing the model(s). Regarding the aviation systems, simulation has been relatively recently applied in a diverse number of cases and problems; in the following section a review is presented.

\section{Literature Review on Airport Modelling}

The studies in airport modelling are diverse. In the following paragraph we present the diverse angles in which some aviation-related problems have been tackled in order to establish why the authors consider the use of simulation as a valid and more powerful tool than the traditional analytical techniques that in most of the cases generate exact solutions.

In literature, one can find many studies that analyse runways systems or allocation problems (check-in or gate allocation), few that include taxiway systems or aprons areas, some others tackle air traffic management policies and operations. In general, most of them are considered isolated problems in which the precedence of processes does not affect the solution, therefore only few analyse all these subsystems in an integral fashion.

Several studies focus on improving the runway throughput due to its importance on the overall airport capacity, for example the studies of Pujet et al. (1999) and Simaiakis and Balakrishnan (2015) uses queue models for increasing capacity, Rathinam et al. (2009) applies dynamic programming for a scheduling problem, Sandberg et al (2014) makes field tests for improving the capacity controlling the pushback rate. Pavlin et al. (2006), put the focus on increasing capacity by ana- lysing the runway occupancy time by making a descriptive analysis of the situation while Burnham et al. (2001) focus on increasing airport capacity of a close parallel runway system by modifying the arrival procedures. On the other hand, Wei and Siyuan (2010), Bazargan et al. (2002), Bertino et al. (2011) present studies using specific-purpose simulators (SIMMOD and TAAM) while Mujica et al. (2017) uses a generalpurpose simulator for including handling operators and aircraft in the same model.

Regarding airport surface management there are relevant work such as the one from Montoya et al. (2010), Simaiakis et al. (2014), and Khadilkar and Balakrishnan (2014), these approaches represent the airside system as a network and then they apply techniques coming from the mathematical programming field to organize the schedule of aircraft.

Other work focuses on how to alleviate congestion problems in airspace and on the ground, especially, they focus on operative problems as the following review illustrates. Concerning problems related to the airspace management, the sequencing and merging or scheduling of arrivals in the terminal manoeuvring area (TMA) has been studied mainly by Beasley et al. (2000), Beasley et al. (2001), Hu and Chen (2005), Michelin et al. (2009), Balakrishnan and Chandran (2010), and Zuniga et al. (2011). Most of the methodologies make an abstract representation of the airspace in order to apply the mathematical optimization techniques for finding efficient solutions to the arrival and departure management.

Studies related to ground operative problems can also be found such as the gate assignment problem presented by Bolat (2000) using a mixed binary math model; Chung and Adeleye (2006) used simulation for the evaluation of turnaround operations, Dorndorf et al. (2007) presents a review of mathematical models in which most of them are exact solutions, Kim and Feron (2012) approach the gate assignment problems using queue models, Mujica Mota (2015) presents a combination of simulation and heuristics for assignment problems in the terminal that can be also implemented in gate assignments or Narciso and Piera (2015) which go further and use a modelling formalism such as coloured Petri nets for gate assignment.

Other researchers have studied the taxi systems such as Mori (2012) whose approach is based on a car traffic congestion model. The research focuses on decreasing the "taxi-out time" and managing the time needed for pushback and engine start. On the other hand, Cetek et al. (2013) uses simulation models for analysing the congestion problem in the manoeuvring areas for identi- 
fying problems of congestion, it uses different runway configurations for identifying potential solutions. Mirkovic (2014) focuses on the study of apron capacity using generic simulation apron models, he calculates apron capacity based on the number of stands and he evaluates different strategies for managing the apron.

Herrera et al. (2014) uses a simulation model for evaluating a new strategy for attending take-off and landings in Mexico City International Airport through the analysis of the air traffic control (ATC) activity. Jacquillat and Odoni, (2015) reported the interdependencies between flight scheduling and airport operations. They proposed a capacity utilization and scheduling model which combines three different models; first, a model of airport congestion that quantifies flight delays as a function of flight schedules and arrival and departure service rates, using a stochastic queuing model; the second model optimizes the sequential control of capacity utilization procedures to minimize congestion costs, for any flight schedule; they use a dynamic programming model. The third one is an integer programming model for scheduling interventions which uses a schedule of flights determined by airline scheduling as its starting point and modifies that schedule in a way that it reduces the imbalances between airport demand and capacity.

As it can be perceived from the review performed, some problems of aviation are tackled with exact methods in which the solution(s) are considered mathematically optimal, however, in most of the cases the solutions might be difficult to be implemented due to the abstraction level in which a minor modification of the situation make the proposed solution unfeasible. In comparison with traditional analytical techniques such as integer and dynamic programming, simulation is a more flexible approach that can be used in a bottom-up fashion, so it is not necessary to oversimplify the description of the system in order to approach it. Furthermore, it can integrate not only all the elements considered in the other techniques such as the causal relationships, but also the uncertainty or variability present in the different processes in the form of probability distributions that capture diverse situations which are used in the model as stochastic variables. For this reason, the authors decided to develop a simulation model that can incorporate the uncertainties of the processes at different levels (strategic and operational) and then evaluate what would be the impact of the proposed implementations with the aggregated effects of the variability present in the system.

In the current paper, we present the methodology for studying a real case study: Mexico City International
Airport. The methodology presented is based on discrete-event systems following a particular one in which we include the strategic uncertainty and stochasticity of the most relevant operational processes in the system to make the simulation as accurate as possible. By using the general-purpose approach, we take advantage of two things; first, we are not limited to the boundaries set by the simulation paradigm of the developers of the available commercial simulators and second we can use the model to scale it up for tackling more complex problems such as the study of the airport network in Mexico (see Wellens and Mujica 2017); this approach could not be possible using a commercial tool as it has been previously discussed. Furthermore, the modelling details are presented in such a way that the reader can replicate the study in a similar airport with a general-purpose tool. We use the methodology for evaluating feasible implementations that can alleviate congestions problems under close-to-reality conditions.

Our work uses a novel methodological approach in the aviation field that can be followed by the reader for evaluating the impact of strategic uncertainty considering also the actual variability of the stochastic processes inherent to the airport. The approach can provide support for airport planners that aim at reducing the risk of over-dimensioning an infrastructure or are interested in finding the practical limitations of growth in a particular airport facility.

\section{Methodological Approach}

A simulation-based approach was used to analyse the performance of the airside of the airport, with the objective of identifying the limitations and bottlenecks during the operation of a high-demand day and for proposing alternatives to alleviate the congestion problems. The model simulates the movements over a 24-hour period; based on a public flight schedule of the airport (OAG 2015). The model was validated using information from Flight Stats and Flight Radar (Flight Radar24 2017); these applications are live representations of the positions and speeds of aircraft worldwide and are based on Automatic Dependant Surveillance Broadcast (ADS-B) which are transponders installed in the aircraft.

The model was composed by putting together the main elements of the airport system, namely: runways, taxiway systems, terminal I and terminal II in the case of Mexico City Airport. The handling operations as well as the modelling of passenger movements were left out of the model. The general approach for the modelling of the system is the one presented in Figure 1. 


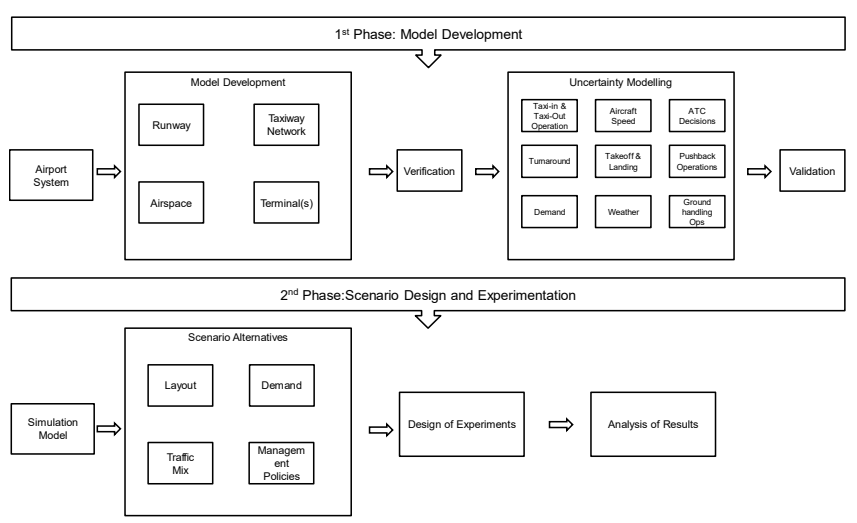

Figure 1: Methodological approach for simulating airport systems.

As one can note, the first phase of the methodology consists of the identification of the elements that should be included in the model, together with the logic and layout. The answer to this question will come from the objective of the study. The next step is to verify the correct logic of the model (verification step). Then, one can decide which elements to include where variability is expected to have more impact in the performance of the system. For the different elements, a different approach can be suggested:

- Taxi Operation: in most of the cases the taxiing speed variability plays an important role in the capacity of the taxi network. It can be modelled as a Uniform probability distribution of speeds or as a Normal or Triangular distribution depending on the data availability.

- Aircraft Speed: the speed has more impact in the modelling of airspace. It can be modelled as a Uniform distribution with the same probabilities for the range of speeds that the different aircraft use based on the radar information.

- ATC: the decision of ATC can be modelled as management policies within the airside, such as the departure priorities. It can be modelled as a scenario using a specific policy for the scheduling of departures or arrivals.

- Turnaround: the turnaround time depend mostly in the type of aircraft and business model of the company (LCCs vs FSCs). It can be modelled as probability distributions that consider the turnaround times of the different combinations Carrier-Aircraft.

- Take-off and landing: It can be modelled as a probability distribution for the runway occupancy times.

- Pushback: It can be modelled as a probability distribution of the delays in pushback start.

- Demand: It can be modelled as different scenarios with different demand levels. This is useful for planning purposes.

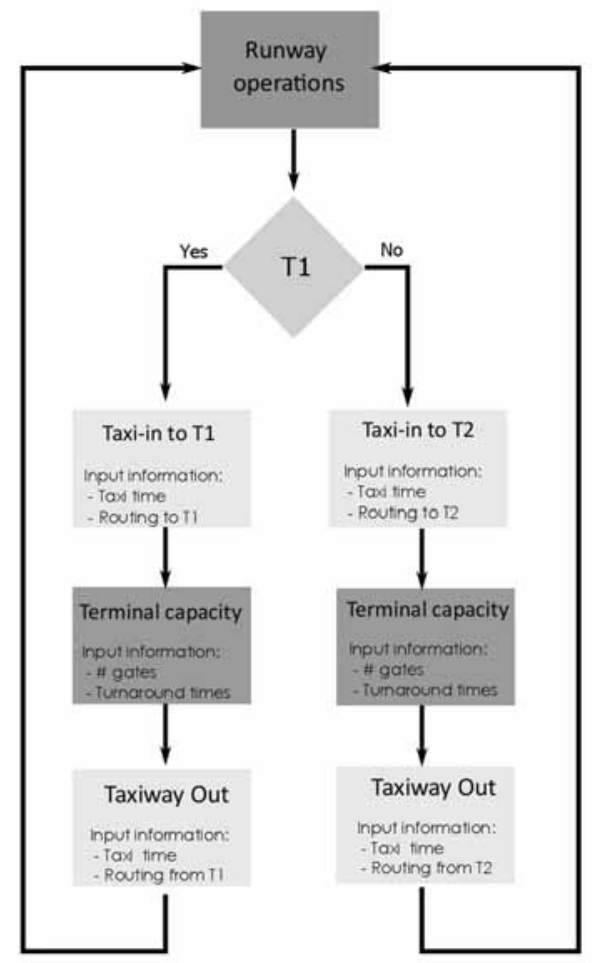

Figure 2: Conceptual approach of the simulation model.

- Weather: this can be modelled as discrete distributions in which the wind direction and strength have a discrete probability of occurrence.

- Ground handling: the operations in ground handling can be modelled as a sequential or parallel probability distributions that are constructed based on the real values of the operations.

For the case under study, the turnaround operation was modelled by a sum of processes represented by delays. The conceptual approach of the model of Mexico is depicted in Figure 2.

Once the model is validated (Sargent 2011), a flight schedule of a representative day has been used as the main input for it. The simulation model allows incorporating the strategic uncertainty or variability of the operations in the airport such as traffic mix, growth, variation in turnaround times, taxi times among others. Furthermore, the level of detail enables the integration of technical and operative restrictions imposed by the airport authority following the International Civil Aviation Organization (ICAO) rules. The most relevant characteristics are the wake-vortex and taxiway separation, taxiway routing for landing and take-off, and the routes followed by the aircraft in the taxiway network and the correspondent speeds during the different considered phases: landing, taxi-in, taxi-out and take off. 
In addition, the aircraft speed, equipment type, and the routing followed by the different aircraft have been also included in the model. In the following paragraphs the characteristics used for the modelling of the different elements of the airport are described in detail.

\section{Case Study: Mexico City International Airport}

In 2015, the Mexican airports network transported more than 73 million passengers and about 655,500 tons of cargo. Regarding the passengers, $63 \%$ were domestic and $37 \%$ international. Mexico City International Airport (IATA code MEX) moved 38.43 million passengers, more than a third of the total traffic of the country (SCT,2015), besides Mexico City being one of the gateways to the country, it is a centralized country which means that the main governmental offices and federal institutions are in Mexico City; for this reason, it is important not to limit the growth and development of the airport in Mexico City. It is forecasted that the demand will continue to grow in the coming years (around $+5 \%$ in aircraft movement and $+12.2 \%$ in number of passengers) and 41 million passengers are expected by 2020 .

\subsection{Airport infrastructure at MEX}

MEX runway system consists of two runways with dimensions of $3963 \times 45 \mathrm{~m}$ and $3985 \times 45 \mathrm{~m}$, respectively. Runways operate in a dependent and segregated mode which means that one is used for arrivals and the other for departures. According to Herrera et al. (2014), the arrivals are performed on the runway $05 \mathrm{R} / 23 \mathrm{~L}$ and departure on runway $05 \mathrm{~L} / 23 \mathrm{R}$. It includes 96 parking positions, 36 direct boarding gates in Terminal 1 (T1), 34 in Terminal 2 (T2), and a total of 74 operative gates. MEX accounts for two passenger terminals, T1 and T2 which are interconnected by a $3 \mathrm{~km}$ monorail and road access. T1 is used for both domestic and international flights and the main partners of SkyTeam for which Aeromexico is part of, whereas T2 is operated mainly by Aeromexico and other airlines. Figure 3 depicts a schematic view of the airside of the airport.

\subsection{Traffic growth in MEX}

According to data from SCT (2015), the biggest domestic airlines in terms of transported passengers are Aeromexico, Volaris, Interjet and Aeromexico-connect. In Mexico, the Low-Cost Carriers (LCCs) business model arrived in 2005 with $5.5 \%$ of the market share and a fast and steady growth allowed them to get to the $60 \%$ of the domestic market by 2013 .

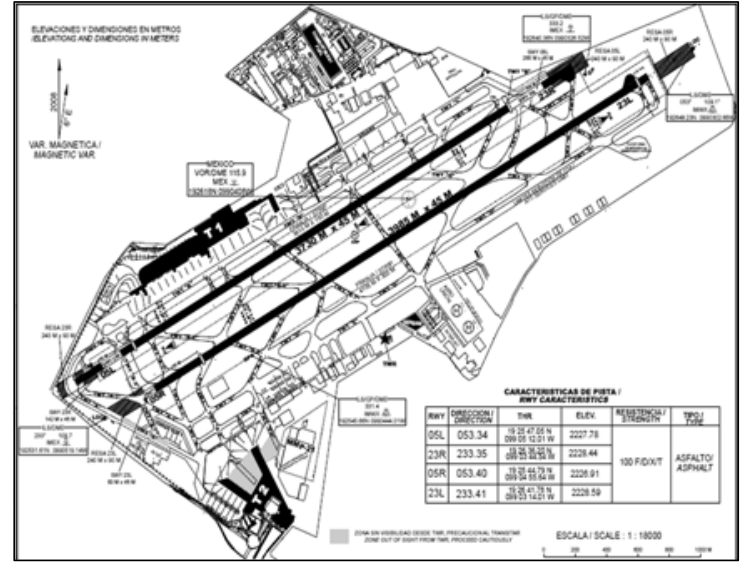

Figure 3: Schematic view of the airport.

This sector is operated by 3 main companies (Interjet, Volaris and VivaAerobus). Unlike these companies, there has been limited development of LCCs, while the potential in the market is clearly there (see Figure 4).

In recent years, LCCs from the US have started operations in MEX, in particular Southwest and JetBlue which is translated into more pressure for the Mexican ATC. Furthermore, it reveals the capacity of LCCs to use their flexible business model for using available slots in congested airports such as MEX in contrast with the Legacy carriers which operate mostly in a HubSpoke basis and require attractive slots so that the feeder traffic can connect with the log-haul flights.

To release the pressure of the current facilities and for allowing the continuous growth of demand, the government has announced the upgrading of a new airport in the vicinity of the current one. This airport will be operational by 2022 in which two runways are expected to be operational. As the previous figures suggest, airlines are interested in increasing their operations in MEX but until the new airport is constructed there is the need of tools that help managers to understand what the true capacity of the current airport is for absorbing more traffic; and they also require tools that provide support in the transition from the operation with the current airport to the operation with the new one.

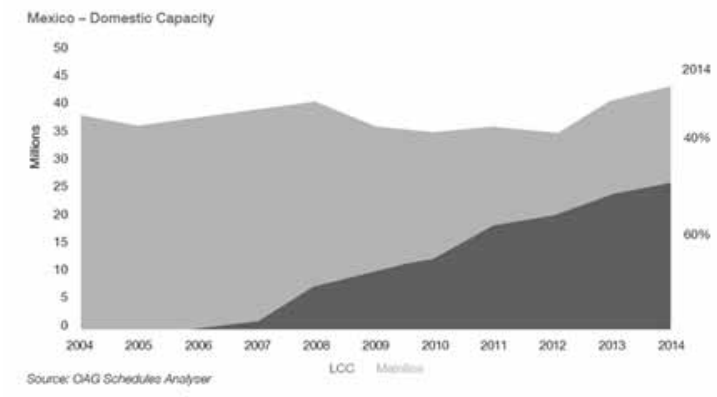

Figure 4: Evolution of traffic on Mex.

SNE 29(1) - 3/2019 
The authors have developed a robust simulationbased methodology that replicates the current operations of the airport combining different models and it is used to get insight about the bottlenecks of the system. It is possible also to understand what the consequences would be in case of the increase in demand and most importantly, it has been used to answer the question if the airport is able to handle more traffic while the new airport is being built.

\subsection{Runway system modelling}

The runway system is composed by two runways separated $330 \mathrm{~m}$. The operation implemented is a segregated one but dependent which means that one runway is used for take off and the other is used for landing, however the separation does not allow using them independently but dependent on each other like in a single runway operation. For the runway operations (landing and take off) there are other characteristics implemented such as runway occupancy time (ROT), landing speeds and restrictions that need to be complied. Due to the lack of information in the schedule, at the beginning of the simulation there are no aircraft parked in the airport, so the simulation starts with empty gates at 5 am which corresponds to the first record of the database. Regarding this assumption, we do not expect that it affects much the analysis, since the airport operates in theory $24 \mathrm{hrs}$ therefore the commercial traffic starts arriving and departing around 5 am when there is enough capacity at the system and it is progressively reduced during the day with the increment in demand.

\subsection{Terminal buildings and turnaround times}

The airport has two terminal buildings (T1 and T2), the first one $\mathrm{T} 1$ is mainly used for international traffic, LCCs and the partners of the SkyTeam alliance for which the flag carrier (AEROMEXICO) is part of. The modelling approach for both terminals consists of a set of available stands where the entities (aircraft) are parked and suffer a delay which simulates the turnaround time (TAT) for each of them. The capacity evaluated of each terminal is the amount of connecting gates which for $\mathrm{T} 1$ is 36 and for $\mathrm{T} 2$ is 34 . The utilization of the terminal building is based on the TATs and the airlines are allocated in accordance with their equipment and company as it is in reality. The turnaround times used are one of the key elements necessary for a correct representation of the operation in the Airport. To construct the probability distributions, we performed data collection and analysis for the different airlines in combination with type of equipment.
The previous data collection was performed extracting information from Flight Radar24 (Flight Radar 2017) and then after pre-processing the data we performed a probability distribution fitting (Banks et al. 1996). In this case, we use an innovative approach by grouping the TATs in pairs of Company-Equipment. This approach captures the effect of the business model of the airline company in the turnaround time of the equipment which is supposed to be high. It differs from other authors such as Herrera et al. (2014) or Wei and Siyuan (2010) that only consider the equipment in their fitting. Table 1 presents the results of the probability distributions used for modelling the TAT.

Since it is unfeasible timewise to extract data for all the airlines and equipment that operate in the airport, we collected information for the companies whose footprint is the biggest in the airport such as Aeromexico-Delta, Volaris, and United. The benefit of using the aforementioned approach is evident in the case of the LCC segment which has very short turnaround times and differ from company to company and differ greatly from the legacy carriers. One can distinguish, and then introduce in the model, the difference of the business models; for example, comparing Interjet A320 and Volaris A320 in which the distributions are different. For those airlines that have less movements in the airport (with the correspondent impact) we assigned a turnaround time similar to the one of an airline for which we collected the data, that is the case of Copa B737-800 and Aeromexico 737800 which use the same distribution.

\subsection{Taxiway system}

The taxiway system was approached as a network of segments interconnected via nodes that enable the aircraft to land and depart using those segments as the taxiways towards and from T1 or T2. Figure 5 illustrates the network developed. The segments are scaled to the real distance they represent; therefore, all the timing calculations are based on Newton's laws taking into consideration length, speed and acceleration of aircraft.

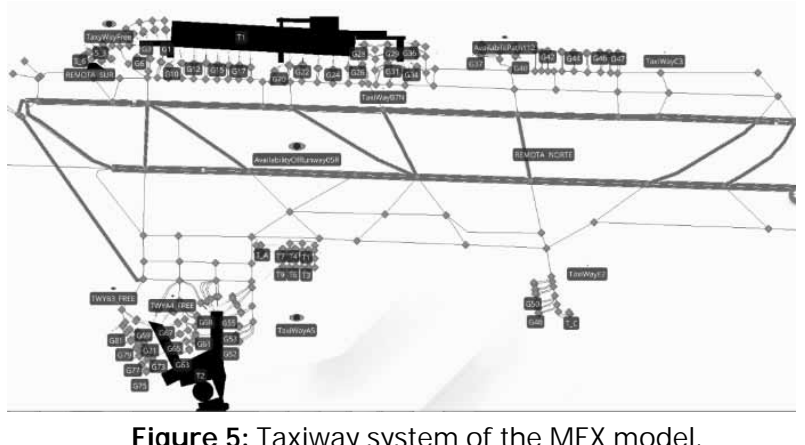

Figure 5: Taxiway system of the MEX model. 


\begin{tabular}{|c|c|}
\hline Airline - Aircraft & $\begin{array}{l}\text { Probability Distribution } \\
\text { (Minutes) }\end{array}$ \\
\hline Aeromexico B787-8 & $(8220+$ Weibull $(4.52,6760)) / 60$ \\
\hline Aeromexico $737-700$ & $(1980+7920 * \operatorname{Beta}(3.18,4.18)) / 60$ \\
\hline Interjet A320 & $(2040+\operatorname{Lognormal}(7.68,0.508)) / 60$ \\
\hline Aeromexico $737-800$ & $\begin{array}{l}(3420+\operatorname{LogLogistic}(3.97, \\
3030)) / 60\end{array}$ \\
\hline VOLARIS A320 & $\begin{array}{l}(4140+\operatorname{LogLogistic}(1.95, \\
1480)) / 60\end{array}$ \\
\hline Aeromexico 777-200 & $(8040+$ Weibull $(1.78,8640)) / 60$ \\
\hline Aeromexico 787-900 & Uniform $(4380,24700) / 60$ \\
\hline AA-B737-800 & $\begin{array}{l}(3420+\log \operatorname{Logistic}(3.97, \\
3030)) / 60\end{array}$ \\
\hline ALL Boeing 747s & $(8040+$ Weibull $(1.78,8640)) / 60$ \\
\hline AEROMAR-ATR & Weibull $(1.100,23.89)$ \\
\hline $\begin{array}{l}\text { Aeromexico } \\
(\text { EMJ,E70,E75,ER4) }\end{array}$ & PearsonVI( $1.065,4.835,209.4)$ \\
\hline Aeromexico-B767-200 & $(8040+$ Weibull $(1.78,8640)) / 60$ \\
\hline Aircanada-A319 & Weibull $(0.8675,46.16)$ \\
\hline Alaska-73H B737-800 & $\begin{array}{l}(3420+\log \operatorname{Logistic}(3.97, \\
3030)) / 60\end{array}$ \\
\hline Avianca Embraer & PearsonVI( $1.065,4.835,209.4)$ \\
\hline Avianca-A319 & Weibull $(0.8675,46.16)$ \\
\hline Avianca-A320 & $(1980+7920 * \operatorname{Beta}(3.18,4.18)) / 60$ \\
\hline Copa B737-800 & $\begin{array}{l}(3420+\operatorname{LogLogistic}(3.97, \\
3030)) / 60\end{array}$ \\
\hline Cubana A320 & $(1980+7920 * \operatorname{Beta}(3.18,4.18)) / 60$ \\
\hline $\begin{array}{l}\text { Delta } \\
\text { B73w(737 winglets) }\end{array}$ & $(1980+7920 * \operatorname{Beta}(3.18,4.18)) / 60$ \\
\hline Delta B757 & $\begin{array}{l}(3420+\log \operatorname{Logistic}(3.97, \\
3030)) / 60\end{array}$ \\
\hline Iberia A345 & Uniform $(4380,24700) / 60$ \\
\hline Interjet SU9 & PearsonVI( $1.129,2.721,129.2)$ \\
\hline LAN B763--767-3 & $(8040+$ Weibull $(1.78,8640)) / 60$ \\
\hline Aeromexico B787-8 & $(8220+$ Weibull $(4.52,6760)) / 60$ \\
\hline Aeromexico B737-700 & $(1980+7920 * \operatorname{Beta}(3.18,4.18)) / 60$ \\
\hline Cargo Flights & Triangular( $(180,420,660)$ \\
\hline UNITED A320 & $(1980+7920 * \operatorname{Beta}(3.18,4.18)) / 60$ \\
\hline UNITED B737 & $\begin{array}{l}(3420+\operatorname{LogLogistic}(3.97, \\
3030)) / 60\end{array}$ \\
\hline UNITED B378 & $\begin{array}{l}(3420+\log \operatorname{Logistic}(3.97, \\
3030)) / 60\end{array}$ \\
\hline UNITED Airlines A319 & Weibull $(0.8675,46.16)$ \\
\hline International- B737s & $\begin{array}{l}(3420+\log \operatorname{Logistic}(3.97, \\
3030)) / 60\end{array}$ \\
\hline InternationalMD83,MD82 & $\begin{array}{l}(3420+\log \operatorname{Logistic}(3.97, \\
3030)) / 60\end{array}$ \\
\hline International - E45X & PearsonVI( $1.065,4.835,209.4)$ \\
\hline International- A319 & Weibull $(0.8675,46.16)$ \\
\hline International- A346 & $(8040+$ Weibull $(1.78,8640)) / 60$ \\
\hline Domestic- B737s & $(1980+7920 * \operatorname{Beta}(3.18,4.18)) / 60$ \\
\hline International - A320s & $(1980+7920 * \operatorname{Beta}(3.18,4.18)) / 60$ \\
\hline International A330 & Uniform $(4380,24700) / 60$ \\
\hline Domestic LCC-A320-A319 & $(2040+\operatorname{Lognormal}(7.68,0.508)) / 60$ \\
\hline Domestic LCC-B733 & $\begin{array}{l}(4140+\operatorname{LogLogistic}(1.95 \\
1480)) / 60\end{array}$ \\
\hline
\end{tabular}

Table 1: Turnaround times for the airlines in the airport.
As it has been mentioned, all the different elements are interconnected with each other following the logic of the airport system. It is important to note that the runway (RWY) system is shared by the two terminals and taxiway systems hence the capacity will be stressed by the utilization of the runway system by the entities going and coming from $\mathrm{T} 1$ and $\mathrm{T} 2$.

\section{Experimental Design: Analysis of Demand}

The experimental design aims at determining what the limitations and bottlenecks of the current system are and what options this system might have for absorbing more traffic. The approach followed first runs replications of the base case scenario with a validated model of MEX (Mendoza et al. 2015). This allows to determine not only the performance indicators but also the impact of the variability present in the system, which in most of cases is due to the turnaround operations and congestion present. Then, three scenarios are evaluated increasing the traffic progressively so that it is possible to determine when the system is not able to absorb the traffic and where the system might collapse.

With the previous approach, we identify if the dynamic of the system behaves linearly with demand or the congestion levels generate such problems in the system that it will be complicated to manage.

Regarding the runway occupancy times and the runway utilization during peak hour, in most of the cases the values were very similar if not identical and since these values do not affect much the congestion levels will not be addressed further in the paper. The main values obtained for the different experiments are presented in Table 2.

\begin{tabular}{|l|l|}
\hline Parameter & Value \\
\hline Runway Occupancy time_Arrival & $40.10 \mathrm{sec}$, STD 1.4 E-14 \\
\hline Runway Occupancy Time Departure & $55.9 \mathrm{sec}$ STD 0.07 \\
\hline RWY Usage (Peak Hour) & $100 \%$ \\
\hline
\end{tabular}

Table 2: Performance parameters of RWY.

\subsection{Base case: current operation}

We will use as input for this base case one of the two busiest day of 2014 (OAG data): $27^{\text {th }}$ of July. For the traffic, we included the cargo movements and the governmental operations which were very active in that day (normally they stand for $10 \%$ of the day movements).

Regarding the actuality of the data, the day selected is considered valid for the analysis of 2017 operations despite it is from 4 years ago. This is due to the fact that it was one of the busiest days of 2014 and assuming the growth since 2013 reported by the Mexican government is correct 
(AICM, 2017), the traffic demand of this day would fall in the first quartile of busy days of the current year.

In this study, we characterize the system using the standard performance indicators of any stochastic system which are normally queuing time, throughput, queue lengths and utilization of resources (Shortle et al. 2017). In aviation systems, the practitioners use their particular names and other similar indicators are used such as On Time Performance, average delay arrival, average delay departure among others, but as for any dynamic system with variability they are correlated with the ones used in the current study. For instance, operational delays are consequence of the queuing times which in turn are a consequence of congestion levels and utilization levels of the resources at the airport. The same applies with the delays in the scheduled flights, due to the congestion levels, the slots are not available when they were originally requested by airlines; thus, by putting attention to the general performance indicators we elaborate our point when comparing one scenario against another. The output indicators provide us with insight about if there is still some spare capacity in the system to accommodate more traffic; and the focus will be put on peak hour-times and throughout the complete day.

For the base case scenario and all the ones performed, we ran 30 replications and obtained the statistics of the performance indicators under study. This is necessary to address the impact of the variability present in the system since it is a stochastic one as it will be illustrated in the correspondent subsections. The base scenario will be detailed so that the reader gets the right understanding of the procedure followed in the study however for the remaining ones we will present only the graphical representation of results which we consider provide more value to the reader about the consequences of the actions taken.

Figure 6 illustrates the evolution of the air traffic movements per hour (ATMs) during the day for one replication while Table 3 presents the performance indicators analysed in the study.

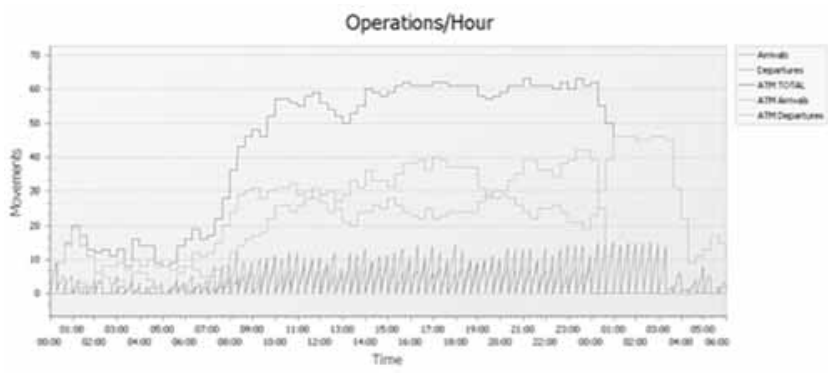

Figure 6: Evolution of ATMs during the day.
In the graph, five indicators are plotted. The first two correspond to the punctual arrivals in a time slot (Arrivals and Departures), the graph called ATM TOTAL corresponds to the rolling ATM/hr calculated from the correspondent plotted time back to one hour; while the ATM Arrivals and ATM Departures are calculated in the same way and they refer to ATM/hr of arrivals and departure respectively. One can appreciate that the ATM indicator (ATM TOTAL) builds up from the morning until it gets to the declared saturation level which occurs around 11:00 hrs in which the limit of 61 $\mathrm{ATM} / \mathrm{hr}$ is almost reached. It can also be appreciated that the saturation continues until approximately 23:00hrs where the arrivals decrease; the movements from 1 am until 5 am correspond to the aircraft that finalize their turnaround in the simulation. From the figure, one can note that during the morning and partially in the evening there is room for allocating more traffic.

The performance indicators of Table 3 characterize the congestion levels of the model under the assumptions and restrictions mentioned. As it was expected, the RWY is the most limited element of the system as the usage percentage reveals. This is a claim given by the airport operator since years ago.

\begin{tabular}{|l|l|}
\hline Parameter & Value \\
\hline ATM/hr (24-hr based) & Mean 38.9, STD 0.17 \\
\hline ATM/hr Arrival (24-hr based) & Mean 19.6, STD 3.6 E-15 \\
\hline T1 Gates usage (24hr-based) & Mean 21.6 (60\%),STD 1.9 \\
\hline T1 Gates usage (Peak Hour) & Mean 27.05 (75\%), STD 2.6 \\
\hline T2 Gates Usage(24hr-based) & Mean 15.1 (44\%), STD 1.3 \\
\hline T2 Gates Usage (Peak hour) & Mean 22.3 (65.6\%), STD 3.1 \\
\hline Time in queue (hr) & MEAN 0.4, STD 0.08 \\
\hline TWY B A/C in queue (24hr-based) & Mean 3.8, STD 0.71 \\
\hline TWY B A/C in Queue (Peak hour) & Mean 1.96, STD 1.04 \\
\hline TW-A A/C in Queue(24hr-based) & Mean 4.6, STD 1.23 \\
\hline TWYA A/C in Queue (Peak hour) & Mean 8.2, STD 3.0 \\
\hline RWY Usage (24hr - based) & $65 \%$ \\
\hline
\end{tabular}

Table 3: Performance Indicators of the Base Case.

In addition, also the $\mathrm{ATM} / \mathrm{hr}$ during the saturated times is around $61 \mathrm{ATM} / \mathrm{hr}$ which is the declared limit for this airport. Another value to note, is the Gate utilization which is $60 \%$ for $\mathrm{T} 1$ and $44 \%$ for $\mathrm{T} 2$ during the day, these values suggest that there is still room during the day for accommodating more aircraft just paying attention to the terminal facilities.

During the simulation runs we identified hotspots in the taxiway system, those are TWY B and TWY A (See Figure 3) coming from $\mathrm{T} 1$ and $\mathrm{T} 2$ respectively. 
Those taxiways are the ones used to get to the head of the runway, therefore when there is high traffic the aircraft queue towards the RWY. As Table 3 shows, sometimes the number of $\mathrm{A} / \mathrm{C}$ queuing is as high as 8.2 in line with a standard deviation of 3 . In other words, during peak hour times the expected amount of aircraft queuing in TWYA in the $95 \%$ of the cases would be between 2.2 and 14.2 aircraft while for TWY B would be between 0 and 4 aircraft. Furthermore, as the reader can note, the range is quite high therefore it makes difficult to predict the length of the queue during peak hour.

It is also relevant to mention that besides the main restricted element (the RWY) the other elements in the system have still some space for absorbing traffic. This will be investigated with the following scenarios.

\subsection{Scenario l: traffic increase from $10 \%$ to $30 \%$ more traffic}

The main assumptions for this scenario are the following:

- The government operations are moved out of the airport. This is an old discussion in Mexico related to the governmental operations, so it leaves room for more commercial traffic which is the objective pursued by developing the new airport.

- When the demand increases, slots will be granted only in the morning and in the evening. We consider that this allocation makes sense since MEX is a Level 3 coordinated airport with high levels of saturation, therefore it is very difficult that slots are released or granted during the busiest period of operation.

- We do not consider the monopolistic behaviour that the legacy carriers might have over the slots since at this point is still difficult to model.

- The type of new traffic allocated was selected randomly. Thus, the TAT of these dummy aircraft will have diverse timings; therefore, we did not make a distinction between legacy or LCC carriers.

This scenario uses as input the original flight data base with a) $10 \%$ more traffic than the current case, b) $20 \%$ more traffic and c) $30 \%$ more traffic. This increase is to evaluate the practical limitations of the system and it does not follow any particular forecast for the following years.

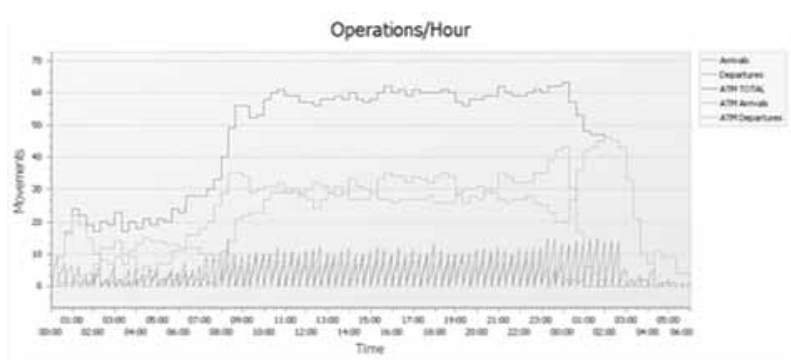

Figure 7: Evolution of ATMs during the day $10 \%$ more traffic.
Regarding the allocation of aircraft, in the case of $10 \%$ increase of traffic, it corresponded to 57 aircraft in the day of operation since we had 589 arrival flights in the data. We allocated 46 flights evenly in the morning from 00:00 to 9:00 which corresponded to 6 more flights every hour ( $+6 \mathrm{ATM} / \mathrm{hr})$ in this period; or one new flight every 11 minutes. For the evening, in the time from 23:00 to 1:00 of the next day we allocated evenly separated the remaining 11 flights. This means approximately an increase of $5 \mathrm{ATM} / \mathrm{hr}$. The traffic was randomly assigned to $\mathrm{T} 1$ and $\mathrm{T} 2$ and the gate used, and TAT was also allocated on a random basis. For the remaining scenarios $(+20 \%$, and $+30 \%)$, the allocation followed the same reasoning, but it changed the number of aircraft to 117 and 176 more flights correspondingly. Figure 7 illustrates how the traffic evolves with 10\% more flights and Figures 8, 9, 10, 11, and 12 present the dependencies of the performance indicators with the increase of traffic.

Figure 7 is similar to Figure 8 but with the increase in $10 \%$ more traffic. The indicators are calculated the same way as explained in the previous sections. As it can be seen, with $10 \%$ increase, the saturation from the morning until the evening is still present as expected, but the morning seems to grow a bit compared with a similar graph from the base case.

In Figure 8, two graphs are presented, the one that plots the average values of the different experiments, and the other that plots the maximum values that were reached during the experiments in particular moments of time (the practical maximum capacity). One appreciates that the average values of the ATM/hr grow linear with the increase of traffic, however the maximum values reached are limited. The limitation is because the levels of congestion limit the maximum values that can be reached as it can be appreciated in the following figures. It is worth noting, that the Max Reached line show that in moments of time, the declared limit of $61 \mathrm{ATM} / \mathrm{hr}$ is surpassed which might cause congestion (and extra ATC workload) in different elements of the system as the following figures confirm.

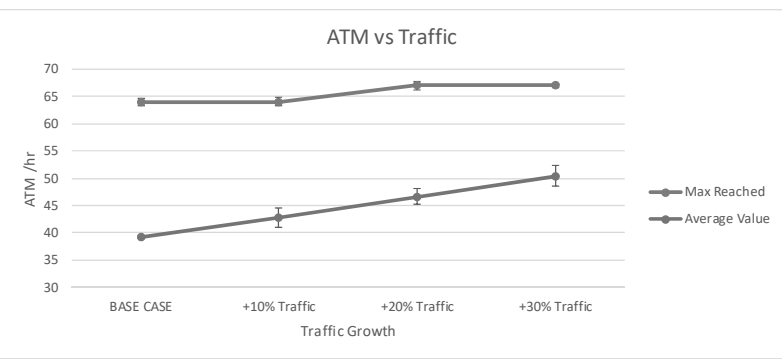

Figure 8: Evolution of ATM/hr vs traffic increase. 


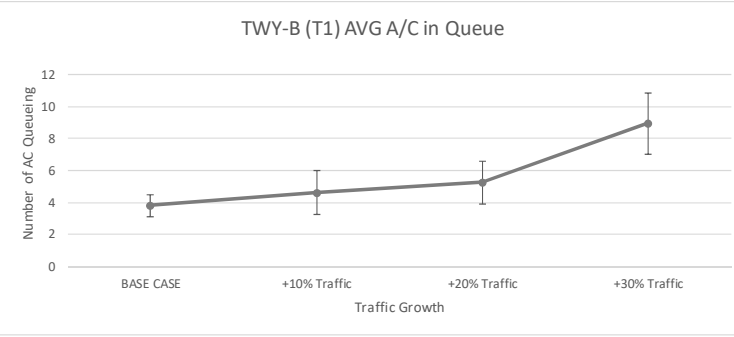

Figure 9: Evolution of $\mathrm{A} / \mathrm{C}$ queuing in $\mathrm{T} 1$.

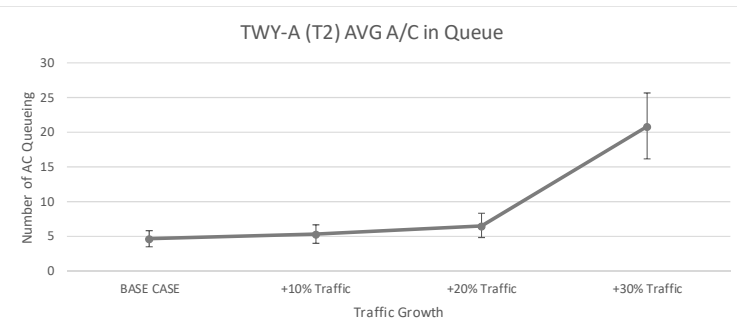

Figure 10: Evolution of $A / C$ queuing in $T 2$.

In Figure 9, it is possible to appreciate that the queue lines grow linear until the $20 \%$ increase, while in the $30 \%$ scenario it grows to 9 aircraft in average as it can be appreciated. Furthermore, the standard deviation increases also representing that in the $95 \%$ of the cases during a $30 \%$ traffic scenario the expected queue will be between 7 and $11 \mathrm{~A} / \mathrm{C}$ coming from T1 to the RWY.

Figure 10 illustrates the effect that demand has in the queue levels of aircraft coming from $\mathrm{T} 2$. In this case, until the $20 \%$ scenario, the number of $\mathrm{A} / \mathrm{C}$ queuing is about 5 with as small variability as can be perceived from the standard deviation line. On the other hand, with the $30 \%$ scenario, the amount of aircraft queuing increases dramatically to a level of 21 with a variability of 5 approximately. This means that for the $+30 \%$ scenario, we might expect to have in the $95 \%$ of the cases queues of 16 to 26 aircraft. These queues will directly impact the expected amount of time waiting for take off as the following figure illustrates.

Figure 11 illustrates the effect in the overall indicator of the time in queues that are expected for the scenarios. Again, until the $20 \%$ growth, the average waiting times might be, in the worst case, around 40 minutes.

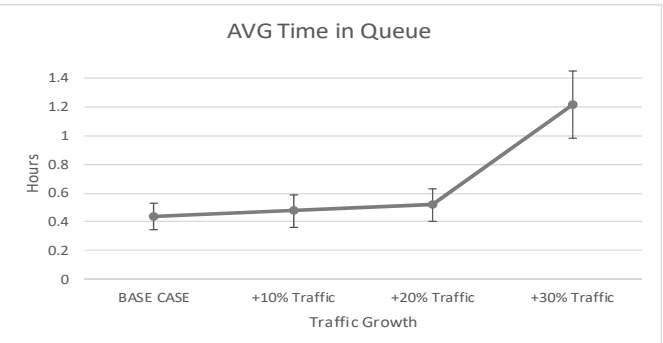

Figure 11: Evolution of average time in queue in the airport.
But in the $30 \%$ scenario the waiting times would increase dramatically that might have to wait between 50 minutes to $1.5 \mathrm{hrs}$ in the $95 \%$ of the cases as the standard deviation lines show.

Regarding the utilization of the gates of the two terminals, the impact is not as with the other performance indicators. Figure 12 illustrates that in general terms, with the increase of demand, the utilization of the gates grows linearly. This behaviour is considered normal, since as with any stochastic system with sequential operations the limiting element is the one that suffers the most and in our case the runway is the one that is hindering the smooth operation of the system.

From Figure 12 it can be appreciated that until the $+20 \%$ scenario $\mathrm{T} 1$ has a utilization of $72 \%$ approximately with some events of $85 \%$ of its capacity. In the case of $\mathrm{T} 2$, the case of the scenario of $+30 \%$, the utilization grows and the variability of the utilization. T1 falls in the interval of $[58 \%, 98 \%]$ in $95 \%$ of the cases while T2 between $65 \%$ and $79 \%$ of its potential. This clearly shows that the limiting factor is the runway and the terminals still have room for absorbing growth in certain moments.

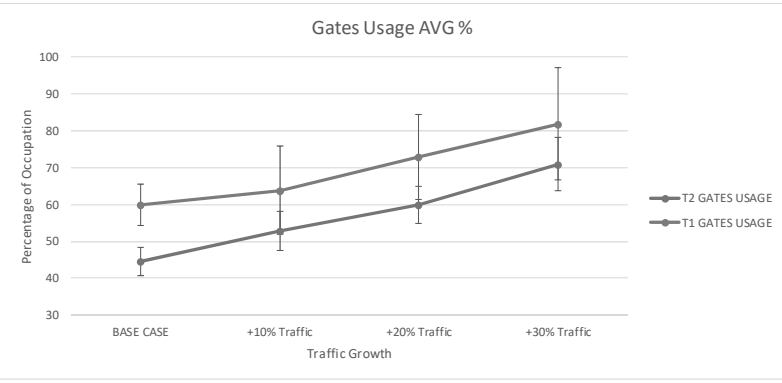

Figure 12: Evolution of gates utilization.

\subsection{Scenario II: increase of traffic as LCCS being the main entrants}

For investigating another situation that might become an alternative to the current situation, we make another extra scenario, one in which we assume that following the trend of the recent years, only Low-Cost Carriers enter to operate at MEX.

For this scenario, the following assumption was taken:

- The increment in traffic continues as it has been since 2006 in which mainly the LCC traffic is the one that grows in the airport (see Figure 12).

The reasoning behind this scenario, is that the LCCs have a more flexible business model and they can operate at slots that would not be attractive to legacy carriers. So, for this scenario, only LCC-type aircraft were added in the model. 
To investigate the impact of this allocation, we simulated the progressive increment of $+10 \%,+20 \%$ and $30 \%$ of traffic. These flights were allocated randomly between both terminals as with the previous scenario; the main difference with the previous one is that the probability distribution that models the TAT is the one used for the LCCs that currently operate in MEX. This probability distribution is the following measured in minutes:

$$
2040+\text { Lognormal }(7.68,0.508)) / 60
$$

Once we run the model with these inputs, we obtained the following graphs which illustrate the impact in the performance indicators for this scenario.

Figure 13 presents the line of Average ATM/hr values and the one of Max ATM/hr reached. In this scenario, it can be appreciated that the average ATM/hr grows linearly with very low variability in the average values; however, in the $+30 \%$ case, the variability makes its presence as it can be seen in the plot of the standard deviation. The other line represents the maximum values reached in the scenarios with the standard deviations representing the variation among experiments of the same scenario. In this case, the maximum values reached are around $64 \mathrm{ATM} / \mathrm{hr}$ however in the last one it increases until approximately $67 \mathrm{ATM} / \mathrm{hr}$ revealing that the system is stressed beyond the declared saturation levels with the correspondent operational problems in the different elements of the system. The following figures illustrate the impact that this increase has in the performance values.

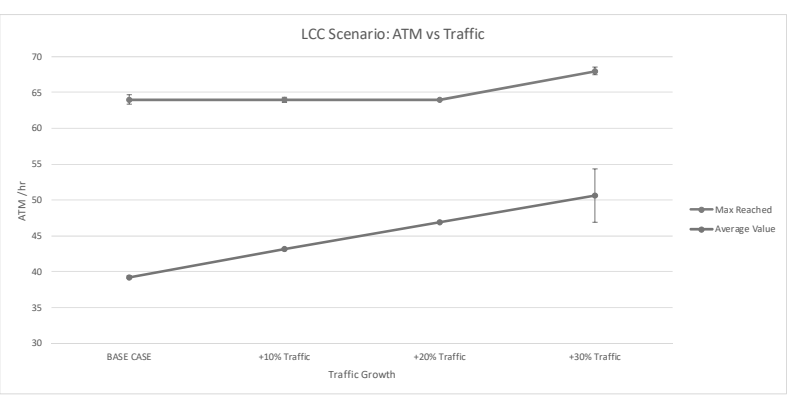

Figure 13: Evolution of ATM/hr.

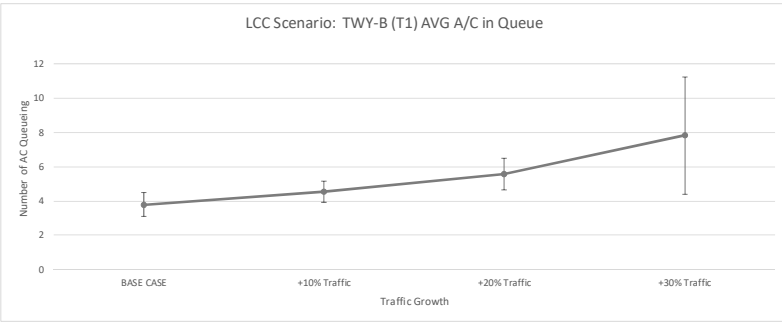

Figure 14: Evolution of aircraft queuing.

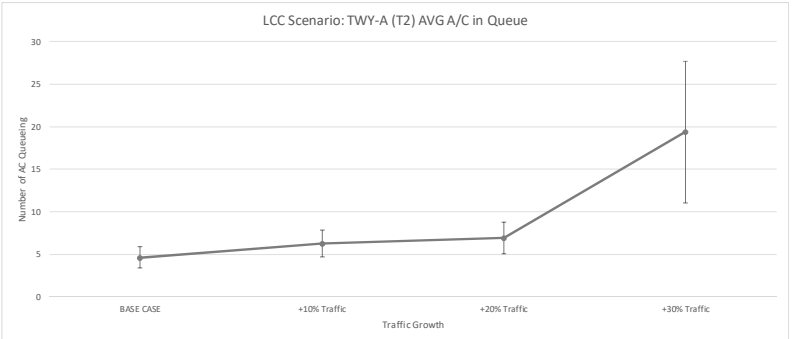

Figure 15: Evolution of aircraft queuing.

As Figure 14 shows, the number of aircraft queuing from T1 to RWY grows linearly with approximately the same variability of $1 \mathrm{~A} / \mathrm{C}$, however in the $+30 \%$ case, the average value goes up but most importantly the variability has increased drastically to $3.5 \mathrm{AC}$ approximately. In other words, in the $+30 \%$ case, $95 \%$ of the time we expect to have between 4.5 to $11.5 \mathrm{~A} / \mathrm{C}$ queuing. In comparison with Scenario I, the numbers are similar, but in this case the variability in the $+30 \%$ case is higher than in the same case of Scenario I.

In this configuration we see a similar behaviour as the one for the queuing at T1. In both cases the variability increases with the last demand number and the queuing size increases but not proportionally. In comparison with Scenario I, the queuing numbers are higher, which is logical, since the type of added traffic makes shorter turnaround times with the consequence of being at the taxi network earlier than in Scenario I.

Figure 16 shows that the time in queue is very similar to the Scenario I, and the graph confirms that with the $+30 \%$ traffic the time waiting is not acceptable for the type of traffic expected to operate at MEX.

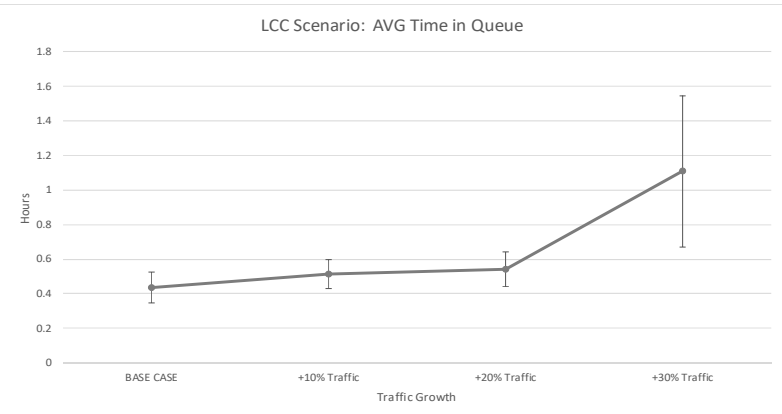

Figure 16: Evolution of time queuing.

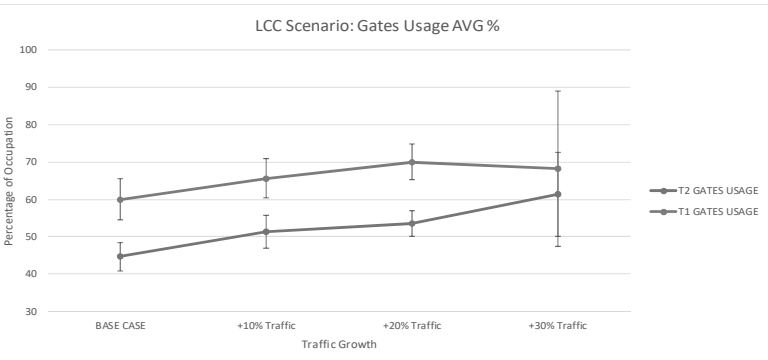

Figure 17: Evolution of gates utilization vs traffic increase. 
Figure 17 illustrates that with the LCCs the gates utilization is less than in the case of Scenario I. For example, in Scenario I, the utilization of the gates sometimes got up to $98 \%$ for T1 while in this scenario T1 does not get to $90 \%$ of utilization and the average utilization is around $70 \%$; this makes a contrast with Scenario I which was approximately $80 \%$ in average. In other words, the utilization of this type of traffic might put more pressure on the runway and taxi system but will release capacity for the gates; therefore, the decision makers need to put attention to the taxi system and runway if the trend continues as it has been in the past years.

\section{Conclusions and Future Work}

The current study presents a methodological approach for analysing capacity limitations in a congested airport. We took the case of International Airpórt of Mexico City as case study. Since in Mexico City, a new airport will be developed in the vicinity for solving congestion problems it is important to have tools that allow decision-makers get insight about the possibilities for the future operation of the current one. The current study analyses the practical limitations of the system using an operative simulation model of the airport system. It aims at evaluating the levels of saturation, since it has been claimed that the airport has reached unbearable levels of saturation. The study demonstrates, that the runway is the main bottleneck of the system but not all the different elements of the system are at the same level of saturation. For this reason, there are still windows of time that can absorb different types of traffic. Taking that into consideration, the simulation model suggests that the current system can absorb more traffic under the current conditions of operation moving out the governmental operations and allocating the traffic early in the morning and in the evening, especially low cost carriers. It is also important to note that due the sequential dependency of the different elements, solving one problem in one element might cause problems to other elements in the system as the second scenario illustrates.

The methodology presented can be used as a blueprint for analysing the current and future limitations of an airport system. As future research we will study the different allocation layouts so that we are able to determine the best progressive implementation of a new facility in combination with the analysis of the TMA which also needs to be considered in order to provide an integral and efficient solution for congested airports.

\section{References}

[1] AICM, 2017, Statistical Information, https://www.aicm.com.mx/categoria/estadisticas.

[2] Airport Research Centre (ARC), 2015. http//www.airportconsultants.com. accessed last time on the 14th august 2017

[3] ATAC's SIMMOD homepage, 2017, http://www.atac.com/simmod-pro.html.

[4] Balakrishnan H, Chandran B. (2010). Algorithms for scheduling runway operations under constrained position shifting. Operation Research, 58 (6), pp.1650-1665.

[5] Banks J, Carson JS, Nelson BL. (1996). Discrete Event System Simulation. 2nd Edition, Prentice Hall.

[6] Bazargan M, Fleming K, Subramanian P. (2002). A simulation study to investigate runway capacity using TAAM. Proceedings of the 2002 Winter Simulation Conference. San Diego, CA. December 8-11, 2002. pp. 1235-1242.

[7] Beasley JE, Krishnamoorthy M, Sharaiha YM, Abramson D. (2000). Scheduling aircraft landings: the static case. Transportation Science, pp.180-197.

[8] Beasley JE, Sonander J, Havelock P. (2001). Scheduling aircraft landings at London Heathrow using a population heuristic. Journal of the Operational Research Society, vol.52, pp.483493.

[9] Bertino J, Boyajian E, Johnson N. (2011). 21st Century Fasttime Airport and Airspace Modeling Analysis with Simmod. Managing the Skies. May/June, pp. 21-23.

[10] Billier B, Nelson B. (2002). Answers to the top ten Input Modeling Questions. Proc. of the 2002 Winter Sim.Conference. December, (San Diego, USA).

[11] Bolat A. (2000). Procedures for providing robust gate assignments for arriving aircraft. European Journal of Operational Research. 120, pp.63-80.

[12] Burnham D, Hallock J, Greene G. (2001). Increasing airport capacity with modified IFR approach procedures for close-spaced parallel runways. Air Traffic Control Quarterly, Vol. 9(1) pp. 45-58.

[13] Cetek C, Cinar E, Aybek F, Cavcar A. (2013). Capacity and delay analysis for airport manoeuvring areas using simulation. Aircraft Engineering and Aerospace Technology: An International Journal, Vol. 86, No. 1, pp. 43-55

[14] Chung C, Adeleye S. (2006). A simulation based approach for contingency planning for aircraft turnaround operation system activities in airline hubs. Journal of Air Transportation, Vol. 11(1)

[15] De Jung F. (2017). The billionaire and the airport: could his last act in Mexico City ruin Carlos Slim?. The guardian, June 28th

[16] DOF (2014). Declaratory: saturation at the Mexico City International Airport. Official Newspaper of the Federation (DOF), Mexico.

[17] Dorndorf U, Drexl A, Nikulin Y, Pesch E. (2007). Flight gate scheduling: State-of-the-art and recent developments. Omega. 35, pp. 326-334.

[18] Flight Radar24 webpage, 2017. https://www.flightradar24.com

[19] Herrera A, Moreno E, Martner C. (2014). Evaluation of a New Strategy for Attending Takeoffs and Landings to Reduce Aircraft Operating Cost and Passenger Delays at the Mexico City International Airport. Engineering Research and Technology, Vol. 15(1), pp. 51-61. 
[20] Hu X, Chen W. (2005). Receding Horizon Control for Aircraft Arrival Sequencing and Scheduling. IEEE Transactions On Intelligent Transportation Systems, vol.6, no.2, pp.189-197

[21] IATA Worldwide Slot Guidelines, 2017, https://www.iata.org/policy/slots/Documents/wsg-8-english.pdf, accessed 29 august 2017

[22] Jacquillat A, Odoni A. (2015). An Integrated Scheduling and Operations Approach to Airport Congestion Mitigation. Operations Research, 63 (6), pp. 1390-1410.

[23] Jensen K, Kristensen LM. (2009). Coloured Petri Nets: Modeling and Validation of Concurrent Systems. Berlin: SpringerVerlag.

[24] Jeppesen's TAAM webpage 2017, ww1.jeppesen.com/industrysolutions/aviation/government/total-airspace-airport-modeler.jsp

[25] Khadilkar H, Balakrishnan H. (2014). Network Congestion Control of Airport Surface Operations. Journal of Guidance, Control, and Dynamics, 37 (3). pp. 933-940.

[26] Kim SH, Feron E. (2012). Impact of gate assignment on gateholding departure control strategies. Proceedings of Digital Avionics Systems Conference (DASC), pp.4E3-1 - 4E3-8, October 14-18, Williamsburg, (Virginia, USA).

[27] Latorre JI, Jimenez E, Perez M. (2013). The optimization problem based on alternatives aggregation Petri nets as models for industrial discrete event systems. SIMULATION, vol.89(3), pp.346-361

[28] Law AM. (2015). Simulation Modeling and Analysis, McGrawHill, 5th edition.

[29] Longo F. (2011). Advances of modeling and simulation in supply chain and industry. SIMULATION, vol.87, pp. 651-656.

[30] Mendoza E, Zuniga C, Mujica M, DelaHaye. (2015). Simulating Airport Capacity: Mexico City Airport Case. In Proc. of ATOS, Delft, The Netherlands.

[31] Michelin A, Idan M, Speyer JL.(2009). Merging of air traffic flows. AIAA Guidance, Navigation, and Control Conference. 10-13 August 2009. Chicago, Illinois.

[32] Mirkovic B, Tošić V, Kanzler P, Hoehenberger M.(2014). Airport Apron Rundabout - Operational Concept and Capacity Evaluation. Proc. of ICRAT 2016, Philadelphia, USA

[33] Montoya J, Woord Z, Rathinam S, Malik W.(2010). A Mixed Integer Linear Program for Solving a Multiple Route Taxi Scheduling Problem. Proceedings of AIAA Guidance, Navigation, and Control Conference. pp. 1-15. August 2-5, Toronto, (Ontario, Canada)

[34] Mori R, (2012). Aircraft Taxiing Model at Congested Airports. 28th International Congress of the Aeronautical Science, 23-28 September, Brisbane, Australia.

[35] Mujica M. (2015). Check-In allocation improvements through the use of a Simulation-Optimization Approach. Transportation Research Part A, pp. 320-335.

[36] Mujica M, Boosten G, De Bock N, Jimenez E, Pinho de Sousa J. (2017). Simulation-based turnaround evaluation for Lelystad Airport. Journal of Air Transport Management, Vol. 64 part A, pp. 21-32.
[37] Narciso ME, Piera MA. (2015). Robust gate assignment procedures from an airport management perspective. Omega. 50. 2015. pp. 82-95.

[38] OECD, 2014 Organisation for Economic Co-operation and Development. Airline Competition DAF/COMP/WD(2014)47

[39] OAG, 2015 https://www.oag.com/

[40] Pavlin S, Zuzic M, Pavicic S. (2006). Runway Occupancy Time as Element of Runway Capacity. Promet - Traffic \& Transportation, Vol. 18, No. 4, pp. 293-299.

[41] Pujet N, Declaire B, Feron E. (1999). Input-output modeling and control of the departure process of congested airports. Proc. of Guidance, Navigation, and Control Conference and Exhibit, pp. 1835-1852, August 9-11, Portland, (Oregon, USA).

[42] Rathinam S, Wood Z, Sridhar B, Jung Y. (2009). A Generalized Dynamic Programming Approach for a Departure Scheduling Problem. AIAA Guidance, Navigation, and Control Conference, pp. 1-12, August 10-13, Chicago, (Illinois, USA).

[43] Rockwell, 2017 https://www.arenasimulation.com/

[44] Sandberg M, Simaiakis I, Balakrishnan H, Reynolds TG, Hansman RJ. (2014). A Decision Support Tool for the Pushback Rate Control of Airport Departures. IEEE Transactions on Human- Machine Systems, 44 (3), pp.416-421.

[45] Sargent RG.(2011). Verification and Validation of Simulation Models. Proc. of 2011 Winter Simulation Conference, (Washington D.C., USA).

[46] SCT, Secretaria de Comunicaciones y Transportes, 2015. Estadística histórica (1992-2014) / Historical statistics (1992-2014).

[47] Shortle J, Thompson J, Gross D, Harris C. (2017). Fundamentals of Queuing Theory. Wiley.

[48] Simaiakis I, Balakrishnan H. (2015). A Queuing Model of the Airport Departure Process. Transportation Science, 50 (1), pp. 94- 109.

[49] Simaiakis I, Khadilkar H, Balakrishnan H, Reynolds TG, Hansman R.J. (2014). Demonstration of reduced airport congestion through pushback rate control. Transportation Research Part A: Policy and Practice. 66, pp.251-267.

[50] Wellens A, Mujica Mota M.(2017). Simulation of the Mexican Airport Network for Addressing a Ground delay program. Proc. Of EMSS 2017, (Barcelona, Spain).

[51] Wei G, Siyuan J. (2010). Simulation study on closely spaced parallel runway analysis using SIMMOD Plus. 2010 International Conference on Intelligent Computation Technology and Automation Proceedings. pp. 344-347.

[52] Yan, S., Shieh C., Chen M. (2002). A simulation framework for evaluating airport gate assignments. Transportation Research Part A, vol. 36, pp.885-898

[53] Zuniga CA, Delahaye D, Piera MA. (2011). Integrating and sequencing flows in terminal manoeuvring area by evolutionary algorithms. IEEE Digital Avionics Systems Conference (DASC), pp.1-11 